\title{
Validation of the modified Japanese Triage and Acuity Scale-based triage system emphasizing the physiologic variables or mechanism of injuries
}

Hiraku Funakoshi ${ }^{1 *}$, Takashi Shiga ${ }^{1}$, Yosuke Homma ${ }^{1}$, Yoshiyuki Nakashima', Jin Takahashi ${ }^{1}$, Hiroshi Kamura ${ }^{1}$ and Masatomi lkusaka

\begin{abstract}
Background: The Canadian Triage and Acuity Scale is a valid triage system. The system was translated and implemented in the Japanese emergency departments (EDs) from 2012. This system was named the Japanese Triage and Acuity Scale; however, the validation studies of the Japanese Triage and Acuity Scale have been limited. In addition, for a patient with multiple complaints, it could become challenging, due to its requirement of a single complaint. Therefore, we hypothesized that a modified version of the Japanese Triage and Acuity Scale using first-order modifiers without chief complaint detection is accurate.

Methods: A retrospective cohort study evaluated a correlation between the modified triage scale level and outcomes of all adult emergency department patients at a Japanese hospital.

Construct validity of the modified triage scale level was assessed based on comparisons of total admission rate (including hospitalizations, emergency department deaths) and length of stay between triage levels.

Results: The distributions of five levels of the triage scale (level 1 is the most urgent) among the 17,121 cases are as follows: 1:451, 2:1148, 3:7703, 4:7652, and 5:167. Total admission rates by each level were 1:89.8, 2:68.2, 3:26.4, 4:6.6, and 5:0.6 \%, which progressively increased from level 5 to 1 and were significant $(p<0.01)$. Compared with patients in level 3, the odds of total admission rates were 14.4, 5.1, 0.27, and 0.030 for the patients in levels 1, 2, 4, and 5 . The length of stay was longer in the patients with the more urgent levels except for those with level 1.

Conclusions: The modified version of the Japanese Triage and Acuity Scale is a valid predictor of total admission and length of stay and may enable the nurses to triage patients without detecting the chief complaints.
\end{abstract}

Keywords: Triage, Patient safety, Canadian Triage and Acuity Scale, Japanese Triage and Acuity Scale

\section{Background}

In the emergency department (ED), a reliable triage system is essential to assess the patients' severity of injury or illness within a short time after their arrival, to assign priorities and to transfer each patient to the appropriate place for treatment [1]. The Canadian Triage and Acuity Scale (CTAS) is one of the most widely accepted web-based triage systems that has

\footnotetext{
* Correspondence: fwhs0754@mb.infoweb.ne.jp

'Department of Emergency Medicine, Tokyobay Urayasu Ichikawa Medical

Center, 3-4-32 Todaijima, Urayasu-shi, Chiba 279-0001, Japan

Full list of author information is available at the end of the article
}

shown high validity and reliability in many research studies in various countries [2-6].

In April 2012, the Japanese Society for Emergency Medicine translated and implemented the CTAS in the Japanese EDs. This system was named the Japanese Triage and Acuity Scale (JTAS). The JTAS has been well accepted in many EDs in Japan. However, the validation studies of the JTAS have been limited, and it has several issues to solve [7].

First, ED overcrowding has been recognized as a major concern leading to a decrease in the quality of care in the EDs. Indeed, two studies have demonstrated an

\section{Springer}


association between ED crowding and increased mortality $[8,9]$. A triage system should not only be valid and reliable but also prompt. From this point of view, there are challenges associated with using the JTAS for triage staff, because the system requires the evaluation of a single chief complaint, even though many patients, especially older patients, have multiple complaints. When a patient is triaged based on the JTAS, the triage nurse initially identifies the patient's single chief complaint. In the second step, the triage nurse determines the triage level, mainly relying on first-order modifiers non-specific to each complaint. Most of the first-order modifiers are common among chief complaints.

Second, the JTAS is notable for its requirement of web connectivity. Due to the limitations of the web connectivity in the Japanese healthcare environment because of guidelines from the Japanese government regarding personal information protection, a triage system based on the JTAS without a web connectivity requirement is beneficial for many Japanese institutions.

The Emergency Severity Index (ESI) is a well-validated triage system that does not require a single chief complaint and is also well validated. Therefore, we examined our hypothesis that the triage level would be accurate when the modifiers were used from the beginning of triage without detecting a single chief complaint.

This is one of the few studies to evaluate the feasibility and validity of a JTAS-based triage system in Japan.

\section{Methods}

\section{Study design, setting, and sample}

We performed a retrospective cohort study of all the adult ED patients who presented to our emergency department (which has 18 ED patient care beds) and were seen by the emergency physicians from April 1, 2013 to March 31, 2014. The study was reviewed and approved by the local Institutional Review Board. The clinical practice committee in our hospital approved implementing this new system because we examined inter-rater reliability of this JTAS-based triage system and presented at the Japanese Society of Emergency Medicine scientific assembly prior to the implementation, and there is no triage system with robust scientific validation in Japan. It was performed at Tokyo Bay Urayasu Ichikawa Medical Center, a 344-bed urban acute care community hospital with an annual ED census of 31,793, in eastern Tokyo, Japan. This is a regional trauma center and designated stroke/cardiovascular center with 24-h capability of percutaneous coronary artery intervention and infusion of t-PA.

All of the adult (18 years old or above) patients treated during the study period seen by board-certified emergency physicians were eligible for inclusion. We excluded all pediatric patients under 18 years of age and adult patients who arrived at the ED to be seen directly by the specialist.

\section{Study protocol}

Table 1 shows our triage system.

We created the modified Japanese Triage and Acuity Scale (mJTAS) according to the original JTAS first-order modifiers and used the mJTAS for the ED referred to in the paper. The mJTAS system comprises eight objective domains, which are the mental status, respiratory status, circulatory status, thermal status, pain status, hemorrhagic status, mechanism of injuries, and Special situation in case of trauma. Each domain has four to five triage levels. The only exceptions to this system are chest pain and hemiparesis, which are always assigned to be level 2 or less. This is because myocardial infarction, cerebral infarction, and cerebral hemorrhage require early intervention, even though patients with those conditions could have normal vital signs. The triage nurse checks each domain and determines the patient triage level based on the domain with the most significant triage level.

We present a typical flow of the mJTAS below. When a 50-year-old male presented with shortness of breath and palpitation to our hospital, our triage nurse measured his vital signs regardless of his complaint. His level of consciousness was E4V5M6 in the Glasgow Coma Scale. His vital signs were respiratory rate $22 / \mathrm{min}$, blood pressure $110 / 70 \mathrm{mmHg}$, heart rate $100 / \mathrm{min}, \mathrm{O}_{2}$ saturation $89 \%$, and temperature $39.0{ }^{\circ} \mathrm{C}$. According to Table 1, level of consciousness was assigned to lowurgency level, breathing was assigned to resuscitation level, circulation was assigned to low-urgency level, and temperature was assigned to emergency level. We determine the triage level based on the highest triage level obtained from all domains. Therefore, his triage level was assigned to the resuscitation level in this case.

The implementation of the mJTAS at the study site began in July 21, 2012. Since then, the mJTAS has been used for every ED patient with no exceptions. There were no occasions that required a change in the mJTAS domains or triage levels or variables. Level 1 patients are the most critically ill and need immediate resuscitation.

The ED nurses were registered nurses who had official training on the measurement of vital signs and/or the level of consciousness, and had standardized training about the mJTAS system prior to the study period.

\section{Study variables}

The variables assessed for a correlation with the patient triage level were the total admission rate (TAR; including hospitalizations, emergency department (ED) deaths, 
Table 1 The mJTAS triage scale. The triage nurse checks each domain and determines the patient's triage level based on the domain with the most significant triage level

\begin{tabular}{|c|c|c|c|c|c|}
\hline Triage level & Resuscitation & Emergency & Urgency & Low urgency & Non-urgency \\
\hline Consciousness & GCS $<9$ & GCS 10-13 & GCS 14 & Alert & \\
\hline Breathing & $\begin{array}{l}\mathrm{SpO}_{2}<90 \% \text { or speak only } \\
\text { single word or upper airway } \\
\text { obstruction }\end{array}$ & $\begin{array}{l}\mathrm{SpO}_{2}<92 \% \text { or labored } \\
\text { breathing }\end{array}$ & $\begin{array}{l}\mathrm{SpO}_{2}<94 \% \text { or } \\
\text { respiratory rate }>22\end{array}$ & $\begin{array}{l}\mathrm{SpO}_{2}>95 \% \\
\text { and breathing normally }\end{array}$ & \\
\hline Circulation & $\begin{array}{l}\text { Shock (systolic blood pressure } \\
\text { under } 80 \mathrm{mmHg} \text { or shock } \\
\text { index (systolic blood pressure/ } \\
\text { heart rate) }<1 \text { ) or weak pulse }\end{array}$ & $\begin{array}{l}\text { Diaphoresis or tachycardia (over } \\
120 \text { beats per minute) or history } \\
\text { of orthostatic hypotension }\end{array}$ & $\begin{array}{l}\text { Apparently abnormal } \\
\text { blood pressure (systolic } \\
\text { blood pressure }>160 \text { or }< \\
100 \mathrm{mmHg} \text { ) }\end{array}$ & Normal blood pressure & \\
\hline $\begin{array}{l}\text { Thermal } \\
\text { status }\end{array}$ & & $\begin{array}{l}\text { SIRS criteria more than } 3 / 4, \\
\text { including a fever higher than } \\
38.5^{\circ} \text { or known to have } \\
\text { neutropenia or a steroid user }\end{array}$ & $\begin{array}{l}\text { SIRS criteria } 2 / 4 \\
\text { including a fever higher } \\
\text { than } 38.5^{\circ}\end{array}$ & Fever only & \\
\hline Pain & & & Pain scale $>8 / 10$ & Pain scale $4-8 / 10$ & \\
\hline Bleeding & & $\begin{array}{l}\text { Active bleeding from head, } \\
\text { neck, or trunk or accompanied } \\
\text { with an open fracture }\end{array}$ & $\begin{array}{l}\text { Epistaxisoractive bleeding } \\
\text { from oral cavity }\end{array}$ & Stopped bleeding & \\
\hline $\begin{array}{l}\text { Mechanism of } \\
\text { injury }\end{array}$ & & $\begin{array}{l}\text { Ejected from a vehicle over } \\
40 \mathrm{~km} / \mathrm{h} \text { or pedestrian hit by a } \\
\text { vehicle or Fall from over } 6 \mathrm{~m} \\
\text { or penetration injury }\end{array}$ & $\begin{array}{l}\text { Motor vehicle accidents } \\
\text { which do not meet the } \\
\text { description within the } \\
\text { left column }\end{array}$ & Bicycle accidents & \\
\hline $\begin{array}{l}\text { Special } \\
\text { situation }\end{array}$ & Cardiopulmonary arrest & Acute chest pain or hemiparesis & & & $\begin{array}{l}\text { Stable } \\
\text { symptom(s) } \\
\text { over } \\
8 \text { weeks }\end{array}$ \\
\hline
\end{tabular}

SIRS systemic inflammatory response syndrome

and transportation to another hospital for admission) and ED length of stay (LOS). The ED LOS was defined as the time in minutes from registration to discharge or admission [10]. These variables were abstracted from electronic medical records. The primary investigator and co-investigators have independently assessed the reliability of the data entry.

\section{Data analysis}

The primary null hypothesis of this study was that there was no correlation between the mJTAS level and the TAR. The chi-square test was performed for categorical variables. In addition, a multivariate logistic regression analysis adjusted for the patient age, sex, and ambulance use was employed to analyze the relationships between the triage level and the rate of total admission. $p$ values were based on a significance level of 0.05 .

For the LOS, the non-parametric Kruskal-Wallis test was used to examine the differences in the ED LOS among triage levels. The raw data for the LOS were reported as medians and interquartile ranges (IQR; 0.25, 0.75). We used a Bonferroni correction to account for the fact that we employed multiple statistical testing; a $p$ value of 0.005 was used to signify statistical significance.
The $95 \%$ confidence intervals $(\mathrm{CI})$ were reported for every result. Statistical calculations were conducted using the STATA (Version 12) software package.

\section{Results}

Patient characteristics and triage level

During the study period, 31,793 patients presented to our emergency department. Out of the eligible patients, 13,485 patients who were under 18 years of age, and 425 patients who arrived at the ED to be seen directly by the specialist were excluded. In addition, 762 were excluded from the data analysis due to missing data (514 patients, $2.9 \%)$ or because the patients were presumed to be outliers due to registration errors (248 patients, $1.4 \%$; a LOS $>720 \mathrm{~min}$ or LOS $<10 \mathrm{~min}$ ). The number of final study samples was 17,121 . Of these cases, the mean age was 50.6 years of age ( \pm standard deviation (SD) \pm 20.7 ), 8858 (51.7 \%) were male, and 6,759 (39.4 \%) were transported by ambulance. The disposition included 13,389 (78.2 \%) discharges, 3481 (20.3\%) admissions, 160 $(0.9 \%)$ cases of transportation to another hospital for admission, and $91(0.5 \%)$ ED deaths. The median LOS was 149.3 (IQR; 10, 717) min.

The distributions of the triage levels among the 17,121 cases were as follows: level 1, 451 (2.6 \%); level 2, 1148 
(6.7 \%); level 3, 7703 (45.0 \%); level 4, 7652 (44.7\%); and level 5, 167 (1.0\%). Table 2 shows the patient characteristics by each triage level.

\section{Disposition}

Table 3 summarizes the details of the disposition by each triage level.

The TARs by each triage level were as follows: level 1 , $89.8 \%$; level 2, $68.2 \%$; level 3, 26.4 \%; level 4, $6.6 \%$; and level $5,0.6 \%$. The TAR progressively increased from triage level 5 to level 1 , and this increase was statistically significant (c2 $=3845$; df $4 ; p<0.001)$.

To analyze the relationship between the mJTAS triage level and the TARs, we developed a logistic regression model. The mJTAS level 3 was used as a reference marker for this evaluation because this was the most common score. Among the triage levels, there were statistically significant differences in that the higher levels were associated with a higher proportion of the total admission, as well as an older age (odds ratio 1.04 $95 \%$ confidence interval (CI) 1.04-1.04), female gender (odds ratio $0.8095 \%$ CI $0.73-0.88$ ), ambulance usage (odds ratio $2.7195 \%$ CI 2.47-2.98), and LOS (odds ratio 1.00 $95 \%$ CI 1.00-1.00). Compared with patients in the mJTAS level 3, the odds ratio for the total admission was 15.1 (95\% CI 10.9-20.9), 5.2 (95\% CI 4.5-6.0), 0.27 (95\% CI 0.25-0.31), and 0.030 (95\% CI 0.005-0.24) for the patients in the mJTAS levels $1,2,4$, and 5 , respectively. The area under the receiver operating characteristic curve for this model was 0.86 .

\section{Length of stay}

The LOS in the ED after registration was longer in the patients with more urgent triage levels, except for the patients with triage level 1 (resuscitation). Figure 1 summarizes the LOS by each triage level. The Kruskal-Wallis test demonstrated statistically significant differences between the LOS based on the triage level $(p<0.001)$.

\section{Discussion}

Our study demonstrated that the triage level assigned by the triage nurses using the mJTAS was strongly associated with the TAR and an increased length of stay in the
ED. As previously mentioned, we demonstrated that the mJTAS had high inter-rater reliability with an intraclass correlation coefficient of 0.77 (Haruka T: Reliability of a CTAS base triage system, unpublished). The reasons for this high inter-rater reliability could be that in this triage system, almost all factors that determine the triage level are objective, such as vital signs or the level of consciousness, and this scale does not require the detection of chief complaints.

The TAR has traditionally been considered an important indicator of severity. Our study confirmed that there is a strong relationship between the mJTAS level and the TAR. However, an admission to hospital is not always a good surrogate marker for severity. For example, patients with a hip fracture are often admitted to the hospital, but their pain status is not significant unless the hip joint is moved, and they often have stable vital signs. In contrast, patients with alcohol intoxication rarely require admission to hospital, although they have a higher level of urgency because they tend to deteriorate quickly or have a low level of consciousness. There are numerous clinical scenarios such as these cases, in which the need for urgent medical assessment may not correlate with hospital admission. However, many studies have adopted an admission to hospital as a surrogate marker $[3-6,11-15]$.

The LOS of patients in the mJTAS level 1 cohort was less than that of patients in the mJTAS level 2 or 3 cohorts. Critically ill patients are associated with relatively simple decisions regarding disposition and are usually transferred to the intensive care unit (ICU). Patients with cardiopulmonary arrest typically receive cardiopulmonary resuscitation for less than $30 \mathrm{~min}$, and once they are resuscitated, they are immediately transported to the ICU. These results are very similar to those described by Dong [2] who reported that the LOSs were $197 \mathrm{~min}$ in the resuscitation category, $351 \mathrm{~min}$ in the emergency category, $309 \mathrm{~min}$ in the urgent category, $206 \mathrm{~min}$ in the low-urgent category and $130 \mathrm{~min}$ in the non-urgent category in patients who were triaged using the CTAS in their ED. Gravel [11], Elshove-Bolk [16], and StormVersloot [10] also reported similar results using different triage systems.

Based on our data using the mJTAS, it could be possible for the hospital to anticipate the necessary inpatient

Table $\mathbf{2}$ The baseline characteristics stratified by the mJTAS level

\begin{tabular}{llllll}
\hline & Resuscitation & Emergency & Urgency & Low urgency & Non-urgency \\
\hline Number of cases & 451 & 1148 & 7703 & 7652 & 167 \\
Mean age $( \pm$ SD) & $68.8( \pm 19.7)$ & $61.1( \pm 20.2)$ & $52.8( \pm 20.7)$ & $45.9( \pm 19.4)$ & $46.9( \pm 19.4)$ \\
Male $(\%)$ & $250(55.4 \%)$ & $671(58.5 \%)$ & $4006(53.0 \%)$ & $3755(49.1 \%)$ & $96(57.5 \%)$ \\
Ambulance use (\%) & $399(88.5 \%)$ & $766(66.7 \%)$ & $3602(46.8 \%)$ & $1988(26.0 \%)$ & $4(2.4 \%)$ \\
\hline
\end{tabular}

SD standard deviation 
Table 3 The details of the disposition stratified by the mJTAS level

\begin{tabular}{lcclll}
\hline & $\begin{array}{l}\text { Resuscitation } \\
(N=451)\end{array}$ & $\begin{array}{l}\text { Emergency } \\
(N=1148)\end{array}$ & $\begin{array}{l}\text { Urgency } \\
(N=7703)\end{array}$ & $\begin{array}{l}\text { Low urgency } \\
(N=7652)\end{array}$ & $\begin{array}{l}\text { Non-urgency } \\
(N=167)\end{array}$ \\
\hline Discharge (\%) & $46(10.2 \%)$ & $365(31.8 \%)$ & $5668(73.6 \%)$ & $7144(93.4 \%)$ & $166(99.4 \%)$ \\
Admission (\%) & $308(68.3 \%)$ & $752(65.5 \%)$ & $1931(25.1 \%)$ & $490(6.4 \%)$ & $1(0.6 \%)$ \\
Transportation to another hospital for admission (\%) & $7(1.6 \%)$ & $30(2.6 \%)$ & $104(1.4 \%)$ & $18(0.2 \%)$ & $0(0 \%)$ \\
Death in ED (\%) & $90(20.0 \%)$ & $1(0.1 \%)$ & $0(0 \%)$ & $0(0 \%)$ & $0(0 \%)$ \\
\hline
\end{tabular}

resources because the mJTAS levels have accurate correlations with the TAR and ED LOS.

As demonstrated, the results of our study are similar to the previous literature on the original version of the CTAS [2-6]. It means that we might be able to bypass the first step of the CTAS, which is the identification of the chief complaint of the patient, thus leading to faster triage and avoiding issues in patients with multiple complaints. In addition, our study results showed comparable validity with a web-based triage system, which may encourage many Japanese hospitals with limited web connectivity due to personal information protection regulation to use a scientifically validated triage system.

\section{Limitation}

Although, the population pyramid of the Urayasu Ichikawa Area is quite similar to the national population pyramid, one of the limitations of our study design was that this study was conducted at the ED of a single community hospital in Japan. This may limit the generalizability of the results, and a larger multi-center study would be needed to confirm the present findings. Second, the emergency physicians at the study site were

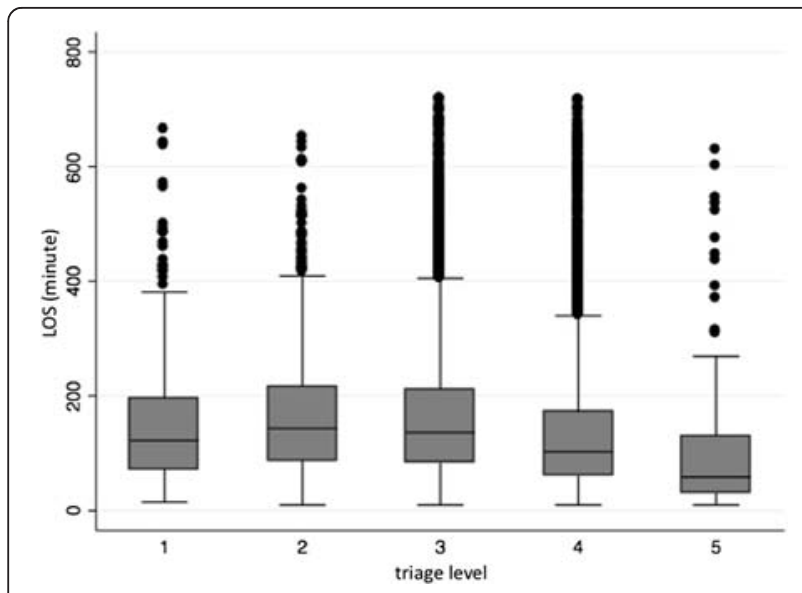

Fig. 1 The length of stay in the ED stratified by the mJTAS level. The box plots indicate the median (horizontal line); the interquartile range (box), not farther away than 1.5 times the interquartile range from the first and third quartiles (whiskers); and the values that are not in the range of whiskers and not considered outliers (dots) not blinded to the triage level, which may have influenced their decision on the disposition. Third, the rate of mistriage is not known. Mistriage could cause data contamination and result in an underestimation or overestimation of the outcome differences. Fourth, our study showed comparable correlation between triage levels and of patients' outcomes such as total admission rate or length of stay with the previous reports of the triage system. However, due to different patient populations between the studies, our study has limitations with regard to its generalizability.

\section{Conclusions}

The modified JTAS triage system is a valid predictor of hospital admission and the ED LOS in the Japanese ED population. The mITAS may enable the nurses to triage patients without detecting the chief complaints. In addition, the mJTAS would be useful for resourcelimited settings, such as in areas where an Internet connection is unavailable.

\section{Abbreviations}

Cl: confidence intervals; CTAS: Canadian Triage and Acuity Scale; ED: emergency department; ICU: intensive care unit; IQR: interquartile ranges; JTAS: Japanese Triage and Acuity Scale; LOS: length of stay; mJTAS: modified JTAS; SD: standard deviation; TAR: total admission rate.

\section{Competing interests}

The authors declare that they have no competing interests.

\section{Authors' contributions}

HF conceived the study. TS and MI supervised the conduct of the study. JT, $\mathrm{YN}$, and HK supervised the data collection. MI managed the quality control. $H F, Y H$, and TS provided statistical advice on the study design and analyzed the data. HF drafted the manuscript, and all authors contributed substantially to its revision. HF takes responsibility for the paper as a whole. All authors read and approved the final manuscript.

\section{Acknowledgements}

We extend our gratitude to our ED members for their dedication and professionalism.

\section{Author details}

'Department of Emergency Medicine, Tokyobay Urayasu Ichikawa Medical Center, 3-4-32 Todaijima, Urayasu-shi, Chiba 279-0001, Japan. ${ }^{2}$ Department of General Medicine, Chiba University Hospital, 1-8-1 Inohana Chuo-ku, Chiba-shi, Chiba 260-8677, Japan. 


\section{References}

1. Christ M, Grossmann F, Winter D, Bingisser R, Platz E. Modern triage in the emergency department. Dtsch Arztebl Int. 2010;107:892-8.

2. Dong SL, Bullard MJ, Meurer DP, Blitz S, Akhmetshin E, Ohinmaa A, et al. Predictive validity of a computerized emergency triage tool. Acad Emerg Med. 2007;14:16-21.

3. Fernandes CMB, McLeod S, Krause J, Shah A, Jewell J, Smith B, et al. Reliability of the Canadian Triage and Acuity Scale: interrater and intrarater agreement from a community and an academic emergency department. CJEM. 2013;15:227-32.

4. Vertesi L. Does the Canadian Emergency Department Triage and Acuity Scale identify non-urgent patients who can be triaged away from the emergency department? CJEM. 2004;6:337-42.

5. Lee JY, Oh SH, Peck EH, Lee JM, Park KN, Kim SH, et al. The validity of the Canadian Triage and Acuity Scale in predicting resource utilization and the need for immediate life-saving interventions in elderly emergency department patients. Scand J Trauma Resusc Emerg Med. 2011;19:68.

6. Jiménez JG, Murray MJ, Beveridge R, Pons JP, Cortés EA, Garrigós JBF, et al. Implementation of the Canadian Emergency Department Triage and Acuity Scale (CTAS) in the Principality of Andorra: can triage parameters serve as emergency department quality indicators? CJEM. 2003;5:315-22.

7. Shimajiri F, Okamoto K, Nishimura A, Imado S, Sawai K, Saitoh I, et al. Evaluation of emergency department triage system in Japan with questionnaires. J J.jn Soc Emerg Med. 2014;15:802-9 (in Japanese).

8. McCarthy ML. Overcrowding in emergency departments and adverse outcomes. BMJ. 2011:342:d2830

9. Guttmann A, Schull MJ, Vermeulen MJ, Stukel TA. Association between waiting times and short term mortality and hospital admission after departure from emergency department: population based cohort study from Ontario, Canada. BMJ. 2011;342:d2983.

10. Storm-Versloot MN, Ubbink DT, Kappelhof J, Luitse JSK. Comparison of an informally structured triage system, the emergency severity index, and the manchester triage system to distinguish patient priority in the emergency department. Acad Emerg Med. 2011;18:822-9.

11. Gravel J, Manzano S, Arsenault M. Validity of the Canadian Paediatric Triage and Acuity Scale in a tertiary care hospital. CJEM. 2009;11:23-8.

12. Pinto D, Salgado P de O, Chianca TCM. Predictive validity of the Manchester Triage System: evaluation of outcomes of patients admitted to an emergency department. Rev Lat Am Enfermagem. 2012;20:1041-7.

13. Jobé J, Ghuysen A, Gérard P, Hartstein G, D'Orio V. Reliability and validity of a new French-language triage algorithm: the ELISA scale. Emerg Med J. 2014:31:115-20.

14. Gräff I, Goldschmidt B, Glien P, Bogdanow M, Fimmers R, Hoeft A, et al. The German version of the Manchester Triage System and its quality criteria_first assessment of validity and reliability. PLoS One. 2014;9:e88995.

15. Dalwai MK, Twomey M, Maikere J, Said S, Wakeel M, Jemmy J-P, et al. Reliability and accuracy of the South African Triage Scale when used by nurses in the emergency department of Timergara Hospital, Pakistan. S Afr Med J. 2014;104:372-5.

16. Elshove-Bolk J, Mencl F, van Rijswijck BTF, Simons MP, van Vugt AB. Validation of the Emergency Severity Index (ESI) in self-referred patients in a European emergency department. Emerg Med J. 2007;24:170-4.

\section{Submit your manuscript to a SpringerOpen ${ }^{\circ}$ journal and benefit from:}

- Convenient online submission

- Rigorous peer review

- Immediate publication on acceptance

- Open access: articles freely available online

- High visibility within the field

- Retaining the copyright to your article 